\title{
Effects of Photobiomodulation Therapy on Glycemic and Lipid Profiles In Vitro
}

\author{
Abdulrahim R. Hakami', Sultan \\ Alasmari', Mohammed Makkawi', \\ Adel Abo Mansour ${ }^{1}$, Irfan Ahmad', \\ Mesfer Al Shahrani', \\ Ali Alkhathami', Ayed A. Dera', \\ Jaya Shanker Tedla², Saleh Almane³, \\ Abdulaziz Abudahish ${ }^{4}$, Jawaher \\ AbuHadhyah ${ }^{4}$, Majed Alshehri ${ }^{5}$, \\ Hanan Abo Mansour ${ }^{6}$, Manar Saad ${ }^{6}$
}

\footnotetext{
${ }^{1}$ Department of Clinical Laboratory Sciences, College of Applied Medical Sciences, King Khalid University, Abha, Asir, Saudi Arabia

${ }^{2}$ Department of Medical Rehabilitation Sciences, College of Applied Medical Sciences, King Khalid University, Abha, Asir, Saudi Arabia

${ }^{3}$ Department of Pathology, Asir Central Hospital, Abha, Asir, Saudi Arabia

${ }^{4}$ Department of Pathology, Maternity and Children Hospital, Abha, Asir, Saudi Arabia ${ }^{5}$ Central Laboratories, King Faisal Medical City (Southern Region), Abha, Asir, Saudi Arabia ${ }^{6}$ Department of Pathology, Armed Forces Hospital Southern Region, Khamis Mushait, Asir, Saudi Arabia
}

Received November 16, 2020

Accepted December 9, 2020

\section{Correspondence}

Abdulrahim R. Hakami

Department of Clinical Laboratory Sciences, College of Applied Medical Sciences, King Khalid University, Abha, Asir, Saudi Arabia

Tel.: +966(0)17241-9268

E-mail: ahakamidakku.edu.sa

(C) Korean Society for Laser Medicine and Surgery

(c) This is an open access article distributed under the terms of the Creative Commons Attribution NonCommercial License (http://creativecommons.org/ licenses/by-nc/4.0) which permits unrestricted noncommercial use, distribution, and reproduction in any medium, provided the original work is properly cited.

\section{Background and Objectives}

This study aimed at examining the effects of photobiomodulation therapy (PBMT) on glucose, cholesterol, triglycerides, and low- and high-density lipoprotein (LDL and HDL, respectively) levels in vitro.

\section{Materials and Methods}

A total of 38 serum samples collected in plain $(n=10)$ and heparinized tubes $(n=28)$ were subjected to PBMT at 60 Joules $(\mathrm{J}) / \mathrm{cm}^{2}$ for 2 min at $810 \mathrm{~nm}$. The glucose and lipid profiles, cholesterol, triglycerides, LDL, and HDL of each sample was measured before and after PBMT.

\section{Results}

A statistically significant increase in glucose levels was observed in the PBMT-sera in 8 out of 10 samples in plain tubes. However, only two samples that were prepared in heparinized tubes showed an increase in glucose levels. The remaining heparinized samples that were exposed to PBMT presented lower glucose values. The treated sera exhibited a fluctuation in the lipid profiles after PBMT. However, high cholesterol levels were evident following PBMT. Similar trends with HDL and LDL in heparinized tubes were evident.

\section{Conclusion}

Together, the findings suggest that photobiomodulation exhibits an effect on glycemic and lipid profiles in vitro. Hence, the use of low-level laser therapy could have therapeutic potential. However, the differences between individual responses appear to indicate that the impact of PBMT may not always be beneficial.

\section{Key words}

Cholesterol; Glucose; Laser therapy; Photo-biomodulation; Triglycerides 


\section{INTRODUCTION}

In the late 1980s, the biological impact of photo-biomodulation therapy (PBMT) was controversial. ${ }^{1}$ This field of research has made obvious progress in recent years. For example, the influence of low-power laser illumination on coronary vessels was used as a potential therapy to prevent restenosis. ${ }^{2}$ In addition, intravascular irradiation with low-energy laser during percutaneous coronary intervention causes a decrease in the magnitude of restenosis and may modulate the inflammatory process in vascular walls. ${ }^{3}$ Therefore, PBMT may be applied in routine clinical practices. Although PBMT has been considered as a safe therapeutic option, ${ }^{4}$ reports of its effects on serum profiles are limited.

Previous studies on PBMT and light-emitting diode (LED) therapy have helped improved our understanding of the underlying mechanisms of PBMT in biological systems. ${ }^{5,6}$ For instance, the main characteristics of photobiomodulation or photo-biostimulation include induction and stimulation of cellular processes. While the specific mechanisms underlying the biological effects of PBMT on cells and tissues remain vague, the response usually exhibits a biphasic dose-response profile. ${ }^{7}$ Moreover, the effect of PBMT on endothelial function and biological molecules have been documented $d^{8-10}$ and have been shown to have proven effects on the lipid composition of cellular membranes ${ }^{11}$ and platelet responses to agonists ${ }^{12}$ In animal models, application of PBMT to bone marrow has been demonstrated to improve angiogenesis and heart function in pigs. ${ }^{13}$

The influence of PBMT on genetic and phenotypic levels is sometimes minimal. A previous study demonstrated no significant alteration in the osteocalcin protein although the time at which the genes are expressed occurred at an earlier phase. ${ }^{14}$ Low-energy laser irradiation caused an increase in nitric oxide (NO) production in some in vivo and in vitro experimental models. Nevertheless, the exact mechanism of this phenomenon is still unknown. ${ }^{15,16}$ In relation to hematological effects, a study reported the effects of low-level laser on reducing whole blood viscosity. ${ }^{17}$ This effect suggests that biological processes are also changed during this process.

Most photo-biomodulation research on diabetes concerned mainly wound healing, and only basic research was done few decades ago about the use of laser as a treatment to diabetes. ${ }^{18-21}$ Although using a laser for this purpose is surely an application with promising results, information regarding the biochemical effects on clinical samples is still lacking. Hence, the aim of this study was to explore the impact of PBMT on serum glucose, triglyceride, cholesterol, and low- and high-density lipoproteins (LDL and HDL, respectively) from selected healthy volunteers.

\section{MATERIALS AND METHODS}

\section{Sample collection}

Blood samples from healthy donors at the Maternity and Children Hospital, Asir Region, Saudi Arabia, who consented to donate blood were chosen for this study. Approximately $5 \mathrm{ml}$ of blood was collected in plain and heparinized tubes. Glucose and lipid profiles from ten samples in plain tubes that were measured. Twenty-eight samples in heparinized tubes were collected on different days; out of these, 12 samples were used for glucose measurements and 16 samples for lipid profile assays. Samples were kept at room temperature for $1 \mathrm{~h}$ and then centrifuged for $5 \mathrm{~min}$ at $3500 \mathrm{rpm}$ to obtain sera. Each sample was separated into two aliquots: (1) without exposure (control samples) and (2) PBMT samples. Serum samples were processed on the same day. We attempted to reduce the time variation during biochemical profile measurement of the two aliquots.

\section{Ethics}

This study was conducted after receiving ethical approval from the Ethics Committee of the Scientific Research, King Khalid University lapproval number: ECM\#2019-103).

\section{Laboratory measurements}

Following serum separation, the test samples were prepared for laser irradiation. To prevent manual positioning error during the laser irradiation, a mechanically adjustable holder for holding the laser probe and maintaining the exact distance was arranged. The laser probe was arranged exactly 90 degrees perpendicular to the sample. For this experiment, we used class 4 Levelaser M1000 Plus (SIMED, Milan, Italy) machine with specifications of $810 \mathrm{~nm}$ wavelength and power generation capability of $1 \mathrm{~W}$ at a frequency of $999 \mathrm{~Hz}^{22}$ The machine was set for 60 Joules $(\mathrm{J}) / \mathrm{cm}^{2}$ for $2 \mathrm{~min}$, and the distance of irradiation was 2 in Table 1. During irradiation, proper precautions, such as wearing gloves and laser safety glasses, were taken. After irradiation, the sample was kept in a separate tray and immediately sent for analysis. Glucose, triglyceride, cholesterol, HDL, and LDL tests were conducted. Every sample was run using the Dimension EXL 200 Integrated Chemistry System ISiemens, 
Munich, Germanyl to perform photometric assays for the quantification of glucose and lipids.

\section{Statistical analysis}

The data are expressed as mean \pm standard deviation. All statistical analyses were performed using GraphPad Software, version 8 (2020) (GraphPad Prism, San Diego, CA, USA). A paired $t$-test was used to calculate the significance of the difference between the control and test patients' samples. A p value of $<0.05$ was considered statistically significant $\left({ }^{*} p<0.05 ;{ }^{* *} p<0.01 ;{ }^{* * *} p<0.001\right)$.

Table 1. Laser therapy parameters used

\begin{tabular}{ll}
\hline Manufacturer & Level Medical \\
Model identifier & Levelaser M1000 Plus \\
Year produced & 2013 \\
Number \& type of emitters (laser or LED) & 1 Laser emitter \\
Wavelength and bandwidth (nm) & $810 \mathrm{~nm}$ \\
Pulse mode (CW or Hz, duty cycle) & $999 \mathrm{~Hz}$ \\
Beam spot size at target $\left(\mathrm{cm}^{2}\right)$ & $1 \mathrm{~cm}^{2}$ \\
Irradiance at target $\left(\mathrm{mW} / \mathrm{cm}^{2}\right)$ & $1000 \mathrm{~mW} / \mathrm{cm}^{2}$ \\
If pulsed, peak irradiance $\left(\mathrm{mW} / \mathrm{cm}^{2}\right)$ & $1000 \mathrm{~mW} / \mathrm{cm}^{2}$ \\
Exposure duration $(\mathrm{sec})$ & $120 \mathrm{sec}$ \\
Radiant exposure $\left(\mathrm{J} / \mathrm{cm}^{2}\right)$ & $60 \mathrm{~J} / \mathrm{cm}^{2}$ \\
Radiant energy $(\mathrm{J})$ & $60 \mathrm{~J}$ \\
Number of points irradiated & 1 \\
Area irradiated $\left(\mathrm{cm}^{2}\right)$ & $1 \mathrm{~cm} 2$ \\
Application technique & Contact less point \\
& application \\
Number and frequency of treatment sessions & $1 \mathrm{session}$ \\
Total radiant energy over entire & $60 \mathrm{~J}$ \\
treatment course $(\mathrm{J})$ & \\
\hline
\end{tabular}

$\mathrm{CW}$, continuous wave.

A

Treated

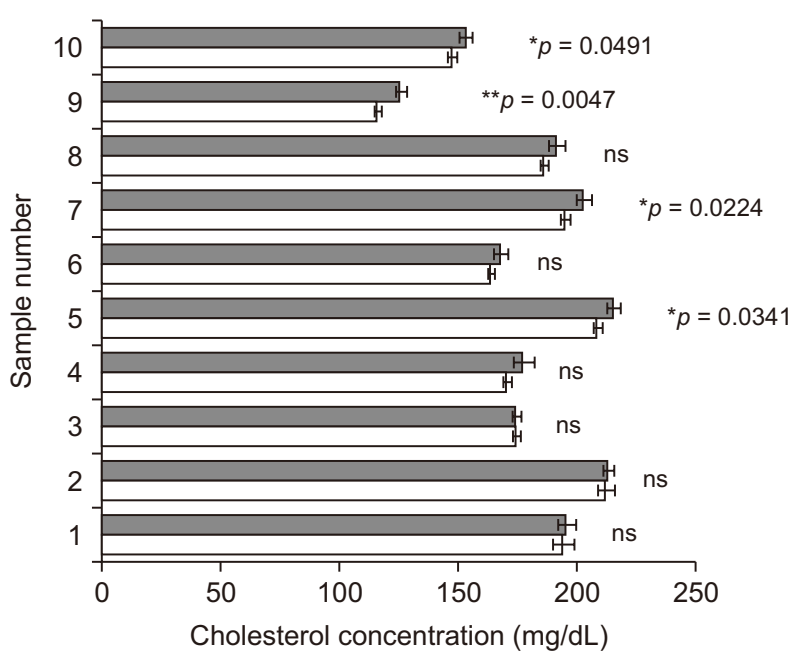

\section{RESULTS}

Preliminary results using plain tubes showed that the biochemistry profile measurements varied between treated and untreated samples and although the differences were not great, further consideration of the differences was worthwhile. Few samples were firstly processed for the determination of cholesterol and triglycerides before and after PBMT. We then increased the sample size to investigate the differences between the laser-treated and the untreated control samples using the paired $t$-test. A

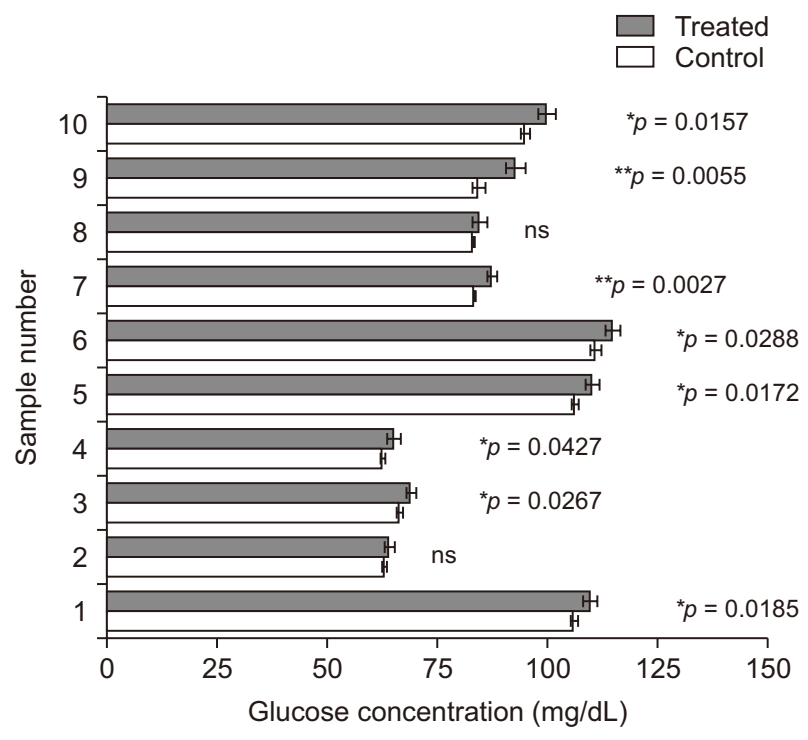

Fig. 1. Glucose concentration before and after PBMT in plain tubes. Comparisons were made using paired t-test. Error bars represent \pm standard deviation $(\mathrm{SD})$ of the means $(\mathrm{n}=3)$.

B

Treated
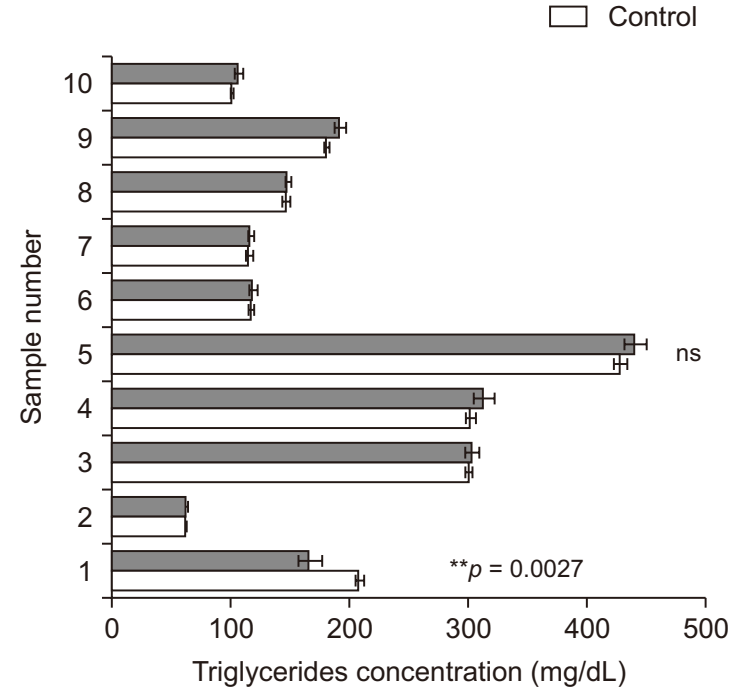

Fig. 2. Cholesterol and triglycerides concentrations before and after PBMT in plain tubes using. Error bars represent \pm SD of the means $(n=3)$. 
fluctuation was found after measuring 38 samples that underwent the same biochemical profile measurement.

\section{Effect on glucose concentration}

We tested glucose (Fig. 1) and lipid profile (Fig. 2) using plain tubes. The tests were repeated using heparinized tubes (Figs. 3 and 4). Glucose concentration in the serum samples was measured in samples with and without PBMT. Two plain tube samples out of 10 showed no significant difference in glucose concentration (Fig. 1). We then repeated the experiment on a different day with new samples using heparinized tubes to prevent glucose utilization and provide reliable data by comparing the two types of collection tubes. With these independent tests, we found that PBMT caused significant reduction in the concentration of glucose in four samples using heparin tubes (Fig. 3). Two out of 12 glucose samples that were separated in heparin tubes showed an increased concen-

Cholesterol (heparinized)

Control Treated

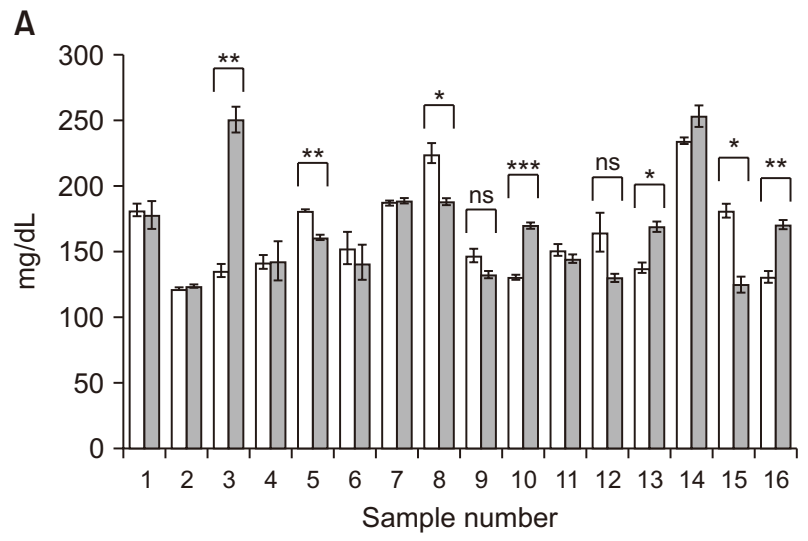

HDL (heparinized)

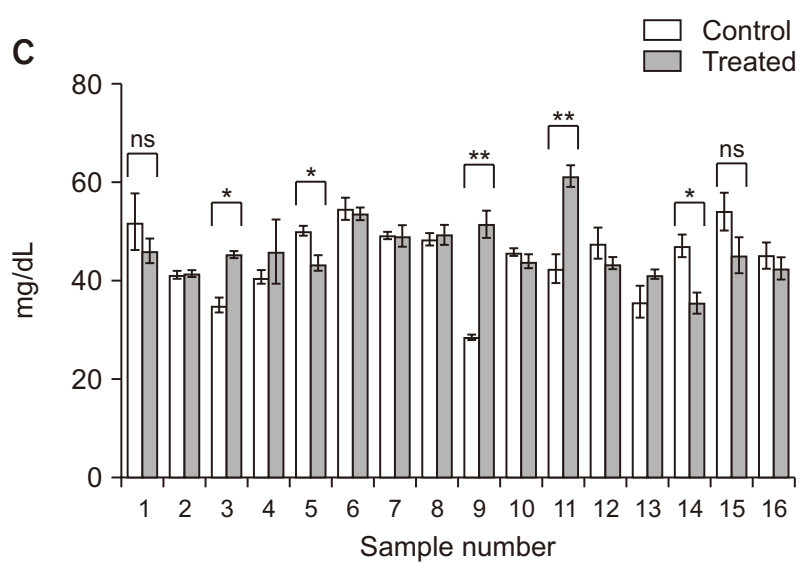

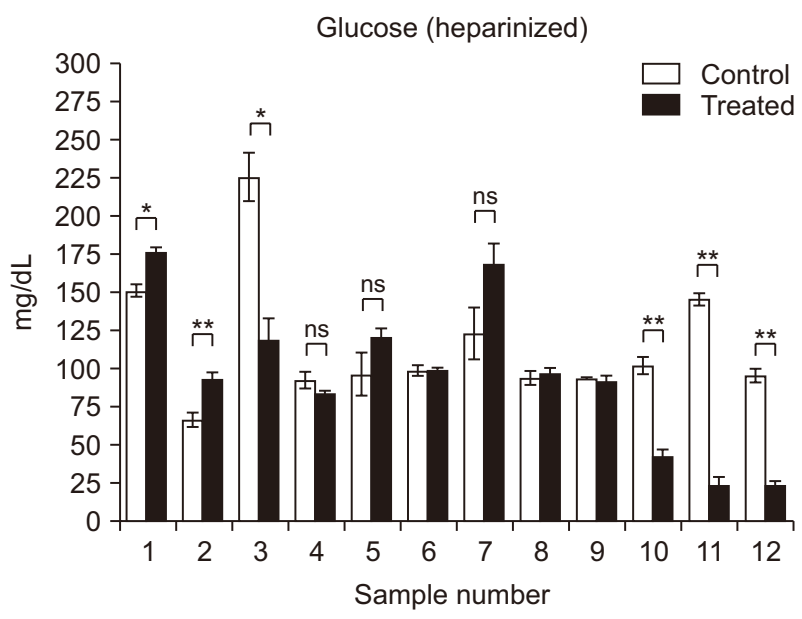

Fig. 3. Glucose concentrations in heparinized tubes. Error bars represent \pm SD of the means $(n=3)$.

Triglycerides (heparinized)

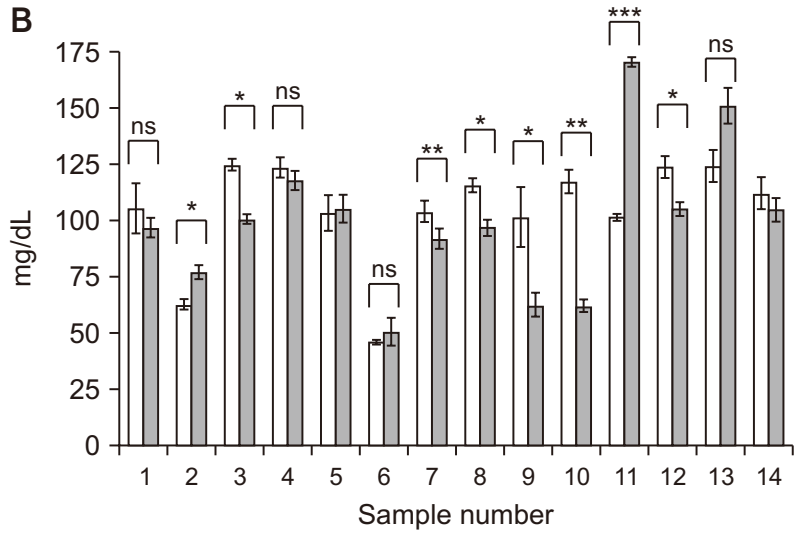

LDL (heparinized)

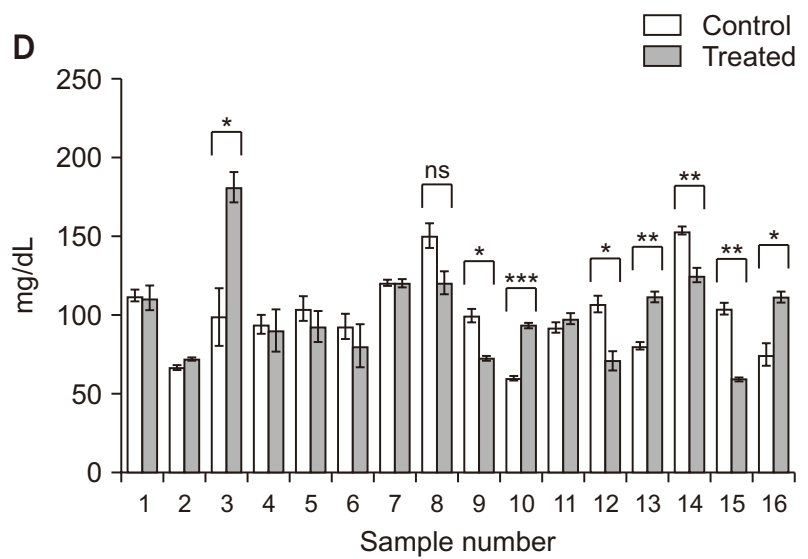

Fig. 4. Concentration of lipid profiles in heparinized tubes. Error bars represent \pm SD of the means $(n=3)$. 
tration following laser therapy.

\section{Effects on lipid profile}

Lipid profiles showed an increase in levels in most of the plain tube samples (Figs. 2 and 4). Interestingly, and unlike glucose, cholesterol levels increased in most plain tube samples following PBMT, but varied levels were observed when samples in heparinized tubes were measured after PBMT exposure. HDL and LDL were included in this study to investigate the behavior of different lipemic profiles due to low-level irradiation. The same trend was found for HDL and LDL in which both presented an upand-down scenario after undergoing PBMT. This finding, along with the results of the glucose test, provide evidence that patients and the biological contents of the samples are determinants of PBMT effects whether by causing an increase or decrease in the biological molecule in question.

Many results matched those generated using heparinized tubes (Fig. 4). However, a fluctuation in triglyceride levels was noted when the test was independently repeated in heparin tubes using new samples (Fig. 4B). It is worth mentioning that PBMT did not have any effects on some serum samples. For example, eight out of $22 \mathrm{glu}$ cose tests did not show significant differences between PBMT-treated and non-treated. The same finding was obtained in 15 out of 24 triglyceride tests, 14 out of 26 cholesterol tests, 11 out of $16 \mathrm{HDL}$ tests, and eight out of 16 LDL tests. Generally, there were 56 tests out of 104 different biochemical tests were shown not to be affected by PBMT.

\section{DISCUSSION}

In this in vitro study, the effect of PBMT on serum biochemical profiles was investigated using serum samples from healthy volunteers and then repeated with independent samples. PBMT did not alter the biochemical molecules in many tested samples. This finding might be interpreted as a safe application, but it should be noted that the effects of this application depend on the patient. All three scenarios have been observed: (1) high, (2) low and (3) no effect. A statistically significant variation was observed in relation to the effect of PBMT on some serum profiles.

Established evidence of the effect of LED therapy on glucose levels exists, ${ }^{23}$ suggesting a significant impact of electromagnetic waves on biologic materials. In our study, low-energy irradiation was used to investigate similar effects on both glucose and lipid profiles. A fluctuation was found in a few biochemical profiles, for example, the effects can cause either an increase or decrease in concentrations of the biochemical substance being tested before and after PBMT, suggesting a different mechanistic effect or due to some variations between samples.

Since a reduction in glucose concentration was noticed after PBMT, comparing the results with results from previously done studies is crucial. The detrimental effect of hyperglycemia on the concentration of cellular adhesion molecules caused by laser irradiation and inflammation reduction after decreasing the soluble fractions of these molecules was previously reported. ${ }^{24}$ In an in vivo study, low-power laser irradiation was shown to decrease hyperglycemia in streptozotocin-induced diabetic rats. ${ }^{25}$ Our findings suggest that in many cases, glucose levels decreased following laser irradiation. In relation to lipid profiles, studies regarding the effect of PBMT on reducing serum lipid levels have also been reported. ${ }^{4}$ A research group reported a positive impact of PBMT on overweight individuals after six weeks of laser treatment when tested twice weekly. ${ }^{26}$ In rats, PBMT was shown to cause a reduction in triglyceride levels, ${ }^{27}$ suggesting a different mechanism of action than in the in vitro model that was generated in some, but not all, samples subject to triglyceride testing. However, some results regarding triglyceride levels were consistent with the results by Aquino et $\mathrm{al}^{27}$ (Fig. 2B, sample 1 and Fig. 4) in which PBMT caused a significant reduction in triglyceride.

Although eight out 16 samples of LDL samples showed no differences between groups, the remaining samples were found to be statistically significant with varying tendencies, suggesting an effect of PBMT on lipids by a mechanism that is yet to be elucidated. Similar varied readings were found with HDL. Moreover, cholesterol levels were higher in treated compared to untreated samples. Eleven cholesterol results out of 26 were significant. All significant differences that were observed in plain tubes showed elevated levels of cholesterol compared to the control samples. Heparin tubes showed three significant results with an increase in cholesterol levels out of four that matched the results of plain tubes. The observation that the biochemical substances decrease upon exposure to low-level laser can be logically interpreted as being due to an increase in energy power associated with the laser and its potential impact on these substances. It is difficult to suggest an explanation for their increases after laser exposure. This finding remains for future studies to solve it.

Furthermore, this study has several limitations. First, the research was limited by the small sample size. Sec- 
ond, this study was also limited by the validity of findings. The measurement differences might be interpreted as negligible. However, these data would provide evidence on the magnitude of their effects on biological molecules. Another notable drawback is that the statistical difference in this experiment can be limited when inter-assay variability is considered although many samples did not show an effect, suggesting that the device has acceptable reproducibility. The differences in this study were from data within the assay. Thus, the use of a larger sample and a much more detailed investigation would improve these types of studies and validate the evidence. Overall, caution is recommended for the clinical use of PBMT or low-level laser irradiation when treating diseases or improving tissue regeneration.

\section{CONCLUSION}

It can be concluded that PBMT at medium power has a tendency to lead to a reduction in biochemical profiles than to increase it. Collectively, the results suggest that low-level laser irradiation or photo-biomodulation therapy exhibits an effect on the glucose and lipid levels, but not in all exposed samples. Additionally, therapeutic potential of using low-level laser was found. However, the impact of laser therapy may not always be beneficial. Compelling evidence from these findings require further studies. Future work can include comprehensive molecular analysis. Having in vitro results regarding photo-biomodulation effects on the glycemic and lipid profiles would help to build cumulative data for future analysis.

\section{CONFLICT OF INTEREST}

The authors declare that they have no competing interests.

\section{REFERENCES}

1. King PR. Low level laser therapy: a review. Laser Med Sci 1989:4:141.

2. Derkacz A, Protasiewicz M, Poreba R, Szuba A, Andrzejak R. Usefulness of intravascular low-power laser illumination in preventing restenosis after percutaneous coronary intervention. Am J Cardiol 2010;106:1113-7.

3. Derkacz A, Protasiewicz M, Poręba R, Doroszko A, Andrzejak R. Effect of the intravascular low energy laser illumination during percutaneous coronary intervention on the inflammatory process in vascular wall. Lasers Med Sci 2013;28:763-8.

4. Avci P, Nyame TT, Gupta GK, Sadasivam M, Hamblin MR. Low- level laser therapy for fat layer reduction: a comprehensive review. Lasers Surg Med 2013;45:349-57.

5. Ferraresi $C$, de Brito Oliveira T, de Oliveira Zafalon L, de Menezes Reiff RB, Baldissera V, de Andrade Perez SE, et al. Effects of low level laser therapy ( $808 \mathrm{~nm}$ ) on physical strength training in humans. Lasers Med Sci 2011;26:349-58.

6. Paolillo FR, Milan JC, Aniceto IV, Barreto SG, Rebelatto JR, Borghi-Silva A, et al. Effects of infrared-LED illumination applied during high-intensity treadmill training in postmenopausal women. Photomed Laser Surg 2011;29:639-45.

7. Huang YY, Sharma SK, Carroll J, Hamblin MR. Biphasic dose response in low level light therapy - an update. Dose Response 2011;9:602-18.

8. Góralczyk K, Szymańska J, Łukowicz M, Drela E, Kotzbach R, Dubiel $M$, et al. Effect of LLLT on endothelial cells culture. Lasers Med Sci 2015;30:273-8.

9. Szymczyszyn A, Doroszko A, Szahidewicz-Krupska E, Rola $P$, Gutherc R, Jasiczek J, et al. Effect of the transdermal lowlevel laser therapy on endothelial function. Lasers Med Sci 2016;31:1301-7.

10. Tsai SR, Hamblin MR. Biological effects and medical applications of infrared radiation. J Photochem Photobiol B 2017;170:197-207.

11. Pasternak K, Wróbel D, Nowacka O, Pieszyński I, Bryszewska M, Kujawa J. The effect of MLS laser radiation on cell lipid membrane. Ann Agric Environ Med 2018;25:108-13.

12. Brill AG, Shenkman B, Brill GE, Tamarin I, Dardik R, Kirichuk VF, et al. Blood irradiation by He-Ne laser induces a decrease in platelet responses to physiological agonists and an increase in platelet cyclic GMP. Platelets 2000;11:87-93.

13. Blatt A, Elbaz-Greener GA, Tuby H, Maltz L, Siman-Tov Y, BenAharon $G$, et al. Low-level laser therapy to the bone marrow reduces scarring and improves heart function post-acute myocardial infarction in the pig. Photomed Laser Surg 2016;34:51624.

14. Bomfim FRCD, Sella VRG, Thomasini RL, Plapler H. Influence of low-level laser irradiation on osteocalcin protein and gene expression in bone tissue1. Acta Cir Bras 2018;33:736-43.

15. Tuby H, Maltz L, Oron U. Modulations of VEGF and iNOS in the rat heart by low level laser therapy are associated with cardioprotection and enhanced angiogenesis. Lasers Surg Med 2006;38:682-8.

16. Chen $\mathrm{CH}$, Hung HS, Hsu SH. Low-energy laser irradiation increases endothelial cell proliferation, migration, and eNOS gene expression possibly via PI3K signal pathway. Lasers Surg Med 2008;40:46-54.

17. Elblbesy MA. Comparative in vitro study: examining $635 \mathrm{~nm}$ laser and $265 \mathrm{~nm}$ ultraviolet interaction with blood. Photobiomodul Photomed Laser Surg 2019;37:342-8.

18. Fridoni M, Kouhkheil R, Abdollhifar MA, Amini A, Ghatrehsa- 
mani M, Ghoreishi SK, et al. Improvement in infected wound healing in type 1 diabetic rat by the synergistic effect of photobiomodulation therapy and conditioned medium. J Cell Biochem 2019;120:9906-16.

19. Forney R, Mauro T. Using lasers in diabetic wound healing. Diabetes Technol Ther 1999;1:189-92.

20. Gogia PP, Hurt BS, Zirn TT. Wound management with whirlpool and infrared cold laser treatment. A clinical report. Phys Ther 1988;68:1239-42.

21. Yu W, Naim JO, Lanzafame RJ. Effects of photostimulation on wound healing in diabetic mice. Lasers Surg Med 1997;20:5663.

22. Jenkins PA, Carroll JD. How to report low-level laser therapy (LLLT)/photomedicine dose and beam parameters in clinical and laboratory studies. Photomed Laser Surg 2011:29:785-7.

23. Francisco CO, Beltrame T, Hughson RL, Milan-Mattos JC, Ferroli-Fabricio AM, Galvão Benze B, et al. Effects of light-emitting diode therapy (LEDT) on cardiopulmonary and hemodynamic adjustments during aerobic exercise and glucose levels in patients with diabetes mellitus: a randomized, crossover, doubleblind and placebo-controlled clinical trial. Complement Ther Med 2019;42:178-83.
24. Góralczyk K, Szymańska J, Gryko Ł, Fisz J, Rość D. Low-level laser irradiation modifies the effect of hyperglycemia on adhesion molecule levels. Lasers Med Sci 2018;33:1521-6.

25. Fukuoka CY, Torres Schröter G, Nicolau J, Simões A. Lowpower laser irradiation in salivary glands reduces glycemia in streptozotocin-induced diabetic female rats. J Biophotonics 2016;9:1246-54.

26. Croghan IT, Hurt RT, Schroeder DR, Fokken SC, Jensen MD, Clark MM, et al. Low-level laser therapy for weight reduction: a randomized pilot study. Lasers Med Sci 2020;35:663-75.

27. Aquino AE Jr, Sene-Fiorese M, Paolillo FR, Duarte FO, Oishi JC, Pena AA Jr, et al. Low-level laser therapy (LLLT) combined with swimming training improved the lipid profile in rats fed with high-fat diet. Lasers Med Sci 2013;28:1271-80.

How to cite this article: Hakami AR, Alasmari S, Makkawi M, Abo Mansour A, Ahmad I, Al Shahrani M, Alkhathami A, Dera AA, Tedla JS, Almane S, Abudahish A, AbuHadhyah J, Alshehri M, Abo Mansour H, Saad M. Effects of photobiomodulation therapy on glycemic and lipid profiles in vitro. MedLasers2020;9:172-178.https://doi.org/10.25289/ML.2020.9.2.172 